\title{
Studies on Various Properties of Diglycine Lithium Sulfate Crystals Grown By Solution Method
}

\author{
G.Emerson Robin ${ }^{1}$, U.Sankar ${ }^{2}$, T.Chithambarathanu ${ }^{1}$, P.Selvarajan ${ }^{3}$ \\ ${ }^{1}$ Physics Research Centre, S.T.Hindu College, Nagercoil, India. \\ ${ }^{2}$ Department of Physics, Sri KGS Arts College, Srivaikundam, India. \\ ${ }^{3}$ Department of Physics, Aditanar College of Arts and Science, Tiruchendur-628216, India.
}

\begin{abstract}
Organic crystals are soft in nature, have low thermal stability, low hardness, low chemical stability and difficult to polish. To overcome these problems, a new class of organic-inorganic hybrid compounds known as semiorganic materials are considered by many researchers. In this work, a semiorganic crystal namely diglycine lithium sulphate was grown and characterized. Single crystals of diglycine lithium sulfate were grown by solution method. The harvested crystals were characterized by solubility studies, XRD studies, SHG studies, microhardness studies, LDT studies, photoconductivity studies and dielectric studies and the results are presented.
\end{abstract}

Keywords: Single crystal, solution growth, NLO material, Charactrization, semiorganic

\section{Introduction}

Inorganic NLO materials possess high melting point, high mechanical strength, and high degree of chemical inertness but poor optical nonlinearity. Organic NLO materials have low melting point, low mechanical strength, high degree of delocalization due to their weak Van der Waal's and hydrogen bondings and also they have the flexibility in the methods of synthesis, scope for altering the properties by functional substitution, inherently high nonlinearity, high laser damage threshold values [1-4]. To consider the advantages of both organic and inorganic NLO materials, researchers concentrate on semiorganic complexes such as Larginine phosphate, L-histidine tetrafluoroborate, L-cystine hydrochloride, L-arginine hydrochloride and the lithium compounds lithium iodate, lithium niobate and lithium tantalate etc [5-11]. Glycine lithium sulfate (GLS) crystals were grown by slow evaporation technique at ambient temperature from aqueous solution of glycine and lithium sulphate taking 1:1 molar ratio [12]. In this work, glycine and lithium sulfate crystals were grown by taking glycine and lithium sulfate in 2:1 molar ratio. The grown crystals of diglycine lithium sulfate were subjected to various characterization techniques like single crystal X-ray diffraction (XRD) technique, Kurtz powder technique, hardness test, second harmonic generation (SHG) test, measurement of laser damage threshold (LDT), dielectric studies and photoconductivity studies.

\section{Solubility and growth}

AR grade chemicals such as glycine and lithium sulfate were purchased commercially. Samples were prepared by taking glycine and lithium sulphate in 2:1 molar ratio. The saturated solution was prepared and stirred well and was filtered and taken in a beaker for crystallization. After a period of 30 days, transparent crystals were harvested. During the growth period, the beaker was covered with perforated polythene paper and the growth vessel was kept in a vibration free platform. Single crystals were grown by slow evaporation technique. Solubility study was carried out using a constant temperature bath (CTB) by gravimetrical method [13]. The salt of the prepared sample was added step by step to $20 \mathrm{ml}$ of double distilled water in an air-tight container kept in the CTB and the stirring was continued till a small precipitate was formed at $30{ }^{\circ} \mathrm{C}$. Then, 5 $\mathrm{ml}$ of the solution was pipetted out and taken in a petri dish and it was warmed up till the solvent was evaporated out. By measuring the amount of salt present in the petri dish, the solubility (in $\mathrm{g} / 100 \mathrm{ml}$ ) of the samples water was determined. The same procedure was followed to find solubility of sample at other temperatures using the constant temperature bath. Fig. 1 shows the solubility curve for diglycine lithium sulfate crystal. From the graph, it is observed that the solubility of the sample in water increases with temperature, exhibiting a high solubility gradient and it has positive temperature coefficient. The Fig. 1 has three regions viz. supersaturated region above the curve, saturated region along the curve and undersaturated region below the curve and the solubility data will be useful to prepare saturated and supersaturated solutions at any temperature in the range $30-50{ }^{\circ} \mathrm{C}$. 


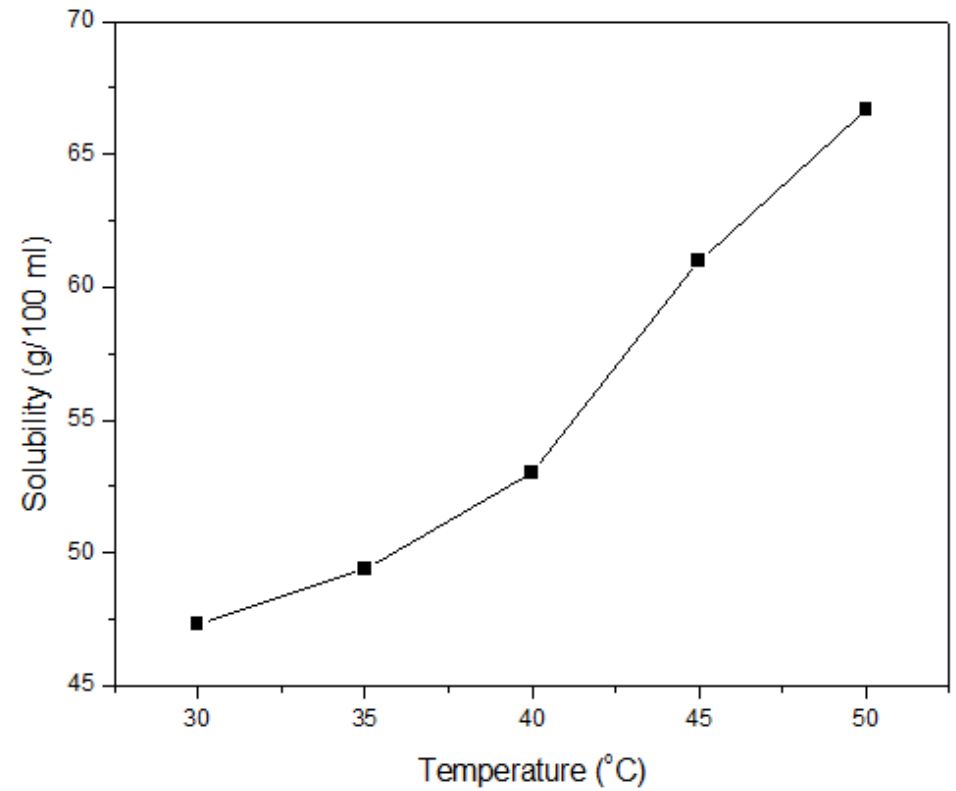

Fig.1: Variation of solubility with temperature for diglycine lithium sulfate crystal

\subsection{Single crystal XRD studies}

\section{Results and Discussion}

The structure of the grown crystals was identified by using a single-crystal X-ray diffractometer (Model: ENRAF NONIUS CAD- $4, \operatorname{MoK}_{\alpha}(\lambda=0.71069 \AA)$ and the lattice parameters were obtained. The obtained data from single crystal XRD studies are given in the table 1 . From the data, it is observed that diglycine lithium sulfate monohydrate crystal belongs to orthorhombic crystal system.

TABLE 1: Crystallographic data for diglycine lithium sulfate crystal

\begin{tabular}{|l|l|}
\hline Diffractometer & ENRAF NONIUS CAD-4 \\
Radiation, wavelength & MoK $_{\alpha}, 0.71069 \AA$ \\
Refinement method & Full matrix Least square method \\
Crystal color & Colorless, transparent \\
Temperature & 293(2) K \\
Symmetry & Orthorhombic \\
a & $8.348(4) \AA$ \\
b & $5.173(3) \AA$ \\
c & $7.834(2) \AA$ \\
$\alpha$ & $90^{\circ}$ \\
$\beta$ & $90^{\circ}$ \\
$\gamma$ & $90^{\circ}$ \\
Volume of the unit cell & $338.31(3) \AA^{3}$ \\
&
\end{tabular}

\subsection{Hardness measurement}

Hardness is an important solid state property and plays a vital role in device fabrication. Hardness, yield strength and stiffness constant are some of the mechanical constants and these properties can be determined by carrying out microhardness studies. Microhardness property was measured using a Vickers microhardness tester, fitted with a Vickers diamond pyramidal indenter. The well polished diglycine lithium sulfate crystal was placed on the platform of Vickers microhardness tester and the loads of different magnitudes were applied in a fixed interval of time. The indentation time was kept $10 \mathrm{~s}$ for all the loads. Vickers microhardness values have been calculated by using the formula $\mathrm{H}_{\mathrm{v}}=1.8544 \mathrm{P} / \mathrm{d}^{2} \mathrm{~kg} / \mathrm{mm}^{2}$ where $\mathrm{H}_{\mathrm{v}}$ is the Vickers microhardness number, $\mathrm{P}$ is the applied load in $\mathrm{kg}, \mathrm{d}$ is the mean diagonal length of the indentation in $\mathrm{mm}$ and 1.8544 is a constant for the geometrical shape of diamond pyramidal indenter [14]. The variation of Vickers hardness number with a load for the grown crystal is shown in the Fig. 2. The result shows that the hardness increases with increase in the load and this can be explained on the basis of depth of penetration of the indenter. When the load increases, a few surface layers are penetrated initially and then inner surface layers are 
penetrated by the indenter with increase in the load. The measured hardness is the characteristics of these layers and the increase in the hardness number is due to the overall effect on the surface and inner layers of the sample and it is called as the reverse indentation size effect.

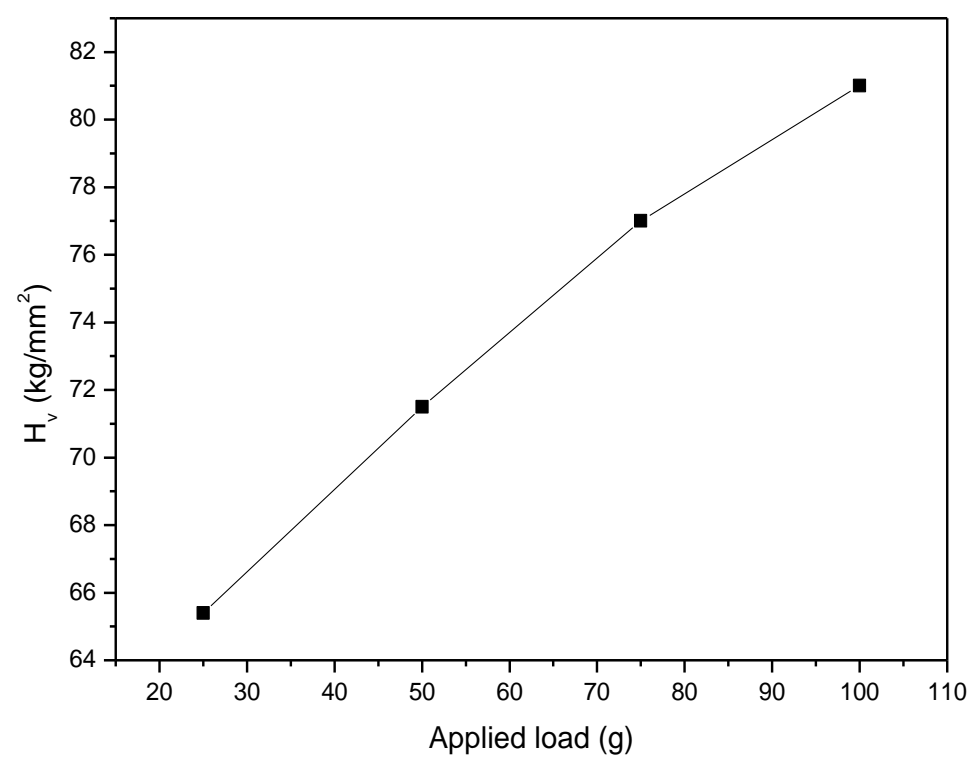

Fig.2: Load dependence of hardness for diglycine lithium sulfate crystal

\subsection{Second-order NLO studies}

One of the second-order NLO studies is Second Harmonic Generation (SHG) test and the sample was tested using the powder technique of Kurtz and Perry [15] using a pulsed Nd:YAG laser(Model: YG501C, $\lambda=1064 \mathrm{~nm}$ ). The grown crystals were ground to powder of grain size of $200 \mu \mathrm{m}$ and the input laser beam was passed through IR reflector and directed on the powdered sample. Potassium Dihydrogen Phosphate (KDP) was used as the reference sample. The SHG behavior was confirmed by the emission of green light $(\lambda=532 \mathrm{~nm})$ from the sample. The measured relative SHG efficiency of diglycine lithium sulfate crystal is 1.63 times that of the standard KDP sample.

\subsection{LDT studies}

Laser damage threshold (LDT) values for the samples were measured using an Nd:YAG laser (1064 $\mathrm{nm}, 18 \mathrm{~ns}$ pulse width). The energy of the laser beam was measured by Coherent energy/power meter (Model No. EPM 200). LDT value is determined using the formula $\mathrm{P}=\mathrm{E} / \tau \pi \mathrm{r}^{2}$ where $\mathrm{E}$ is the energy in mJ, $\mathrm{r}$ is radius of the spot in $\mathrm{mm}$ and $\tau$ is the pulse width. The obtained value of LDT of the grown diglycine lithium sulfate crystal is $0.831 \mathrm{GW} / \mathrm{cm}^{2}$ and the and the higher value of LDT could be useful for manufacturing nonlinear optical devices.

\subsection{Photoconductivity studies}

Photoconductivity is due to increase in the concentration of charge carriers when UV or visible light is incident on a crystalline sample. Photoconductivity studies have been carried out using a photoconductivity set-up with a Keithley 485 picoammeter at room temperature. By connecting the sample in series to a DC power supply and a picoammeter, the dark conductivity of the sample was studied. Electrical contacts were made on the samples using the silver paint. In order to measure the photo current, the sample was illuminated with a halogen lamp by focusing a spot of light on the sample with the help of a convex lens. Photocurrents and dark currents were measured for the sample at different applied DC voltages. The variations of dark and photo currents for diglycine lithium sulfate crystal are shown the Fig.3. It is noticed that the values of dark current and photo current increase with increase of applied electric field. For the sample, it is observed that the photo current is more than dark current and hence the sample has positive photoconductivity. 


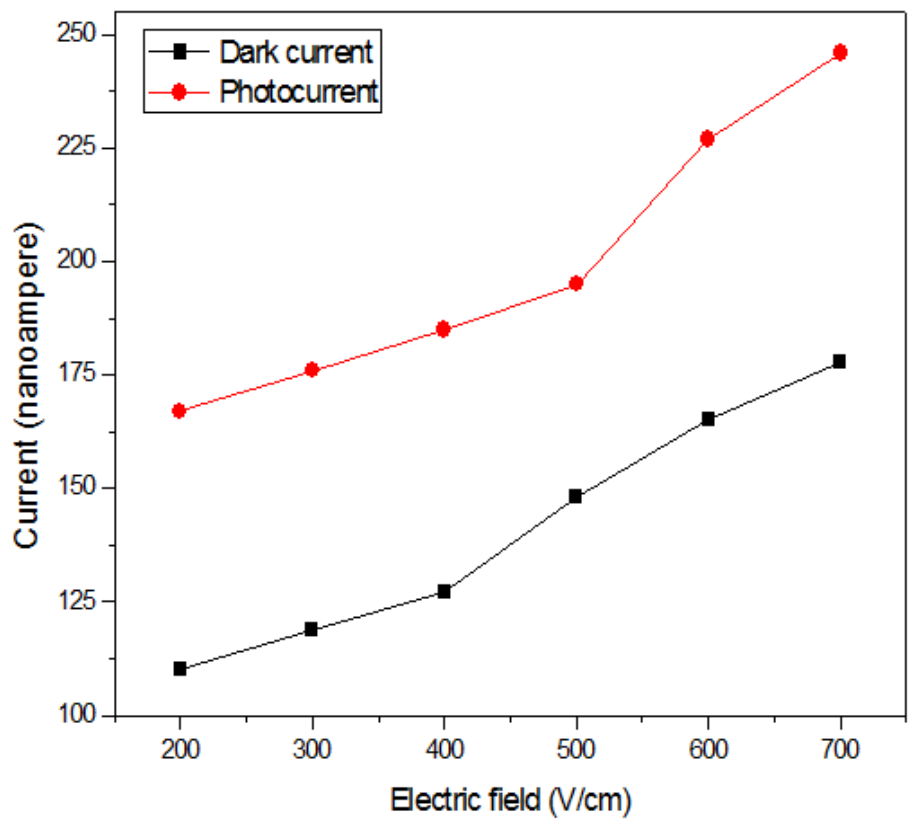

Fig.3: Variations of photocurrent and dark current with the applied field for

Diglycine Lithium Sulfate crystal

\subsection{Dielectric studies}

The capacitance and dielectric loss factor $(\tan \delta$ ) measurements were carried out using the parallel plate capacitor method at a temperature of $30{ }^{\circ} \mathrm{C}$ using an Agilent 4284A LCR meter at different frequencies ranging from $10^{2}$ to $10^{6} \mathrm{~Hz}$. Using the values of capacitance without sample and with sample of the capacitor, the dielectric constant is calculated. The dielectric loss factor can be measured directly from the LCR meter. The variations of dielectric constant and dielectric loss with frequency for diglycine lithium sulfate crystal are displayed in Figs. 4 and 5. It is observed that the dielectric constant has higher values at lower frequencies and further it decreases with increase in frequency and become independent at higher frequencies. The dielectric constant of the materials is due to the contribution of electronic, ionic, dipolar or orientation and a space charge polarization which is high relay upon on the frequencies. The space charge polarization is generally active at lower frequencies. It is observed that the dielectric loss decreases with the increasing of frequency. The low value of dielectric loss with high frequency for diglycine lithium sulfate crystal suggests that the sample possess enhanced quality with lesser defects. Lower values of dielectric constant will increase the efficiency of SHG and this is called as the Miller's rule [16].

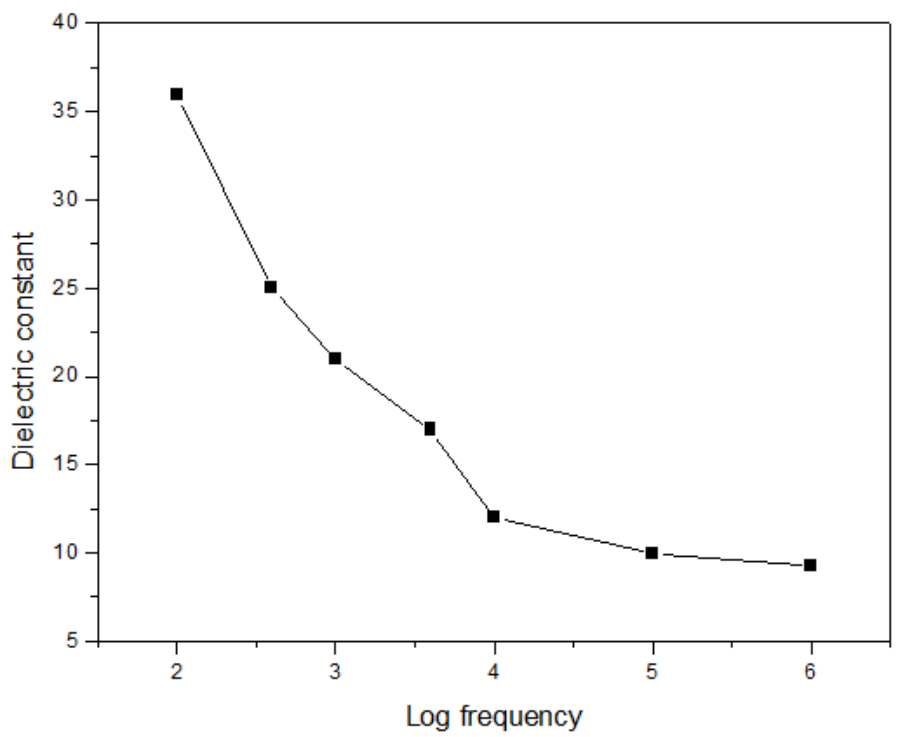

Fig.4: Variation of dielectric constant with frequency for Diglycine Lithium Sulfate crystal at room temperature 


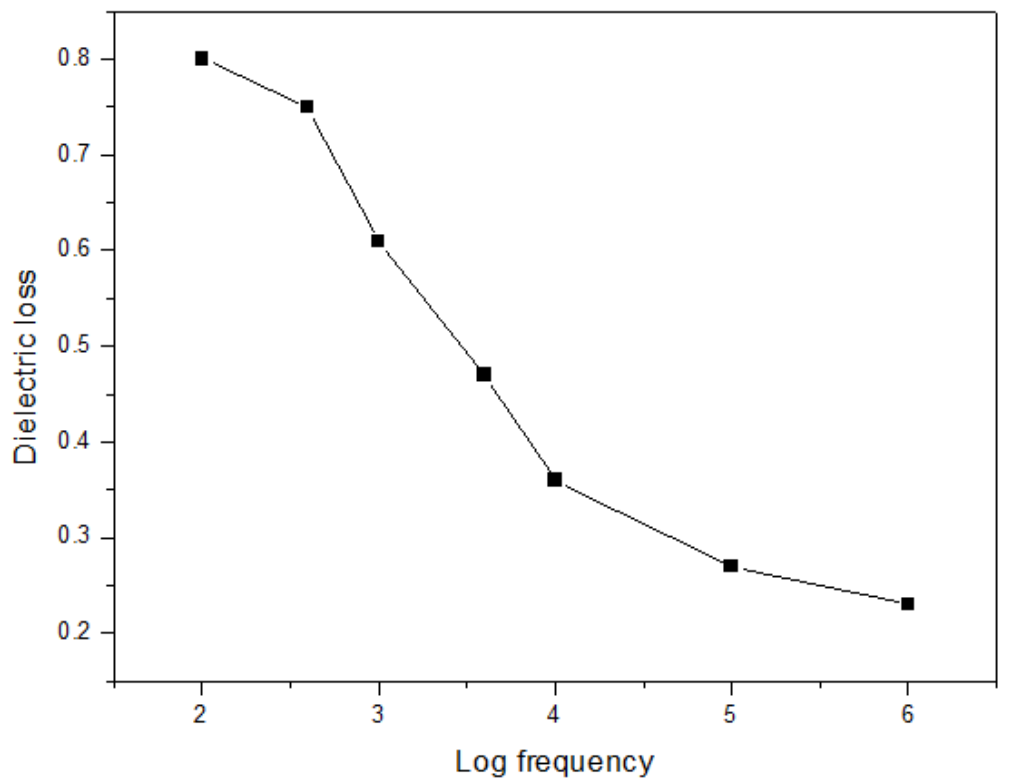

Fig.5: Variation of dielectric loss with frequency for Diglycine Lithium Sulfate crystal at room temperature

\section{Conclusion}

Using the commercially available glycine and lithium sulfate salts, single crystals of diglycine lithium sulfate were grown by slow evaporation technique at room temperature. The solubility of the sample is observed to be increasing with increase in temperature and hence it has positive temperature coefficient of solubility. The crystal structure of the sample is found to be orthorhombic. SHG efficiency of the sample is found to be 1.63 times that of KDP sample. Vickers microhardness studies were carried out for the grown crystal to determine values of hardness. The LDT value of the grown sample was found to be $0.831 \mathrm{GW} / \mathrm{cm}^{2}$. The low values of dielectric constant and loss factor, high values of SHG and LDT of diglycine lithium sulfate crystal ascertain that the sample could be a potential nonlinear optical material for laser applications.

\section{Acknowledgement}

The authors are thankful to the management of KGS Arts College, Srivaikundam, S.T. Hindu College, Nagercoil and Aditanar College of Arts and Science, Tiruchendur for the encouragement given to us to carry out the research work. The authors are grateful to the research staff members of St.Joseph's College, Trichy, STIC, Cochin University, Cresent Engineering College, Chennai, VIT, Vellore, S.T. Hindu College, Nagercoil for taking the research data for our sample.

\section{References}

[1]. C. Krishnan a, P. Selvarajan b, S. Pari, Current Applied Physics 10 (2010) 664-669.

[2]. K. J. Arun, S. Jayalakshmi , J. Min. Mater. Charact. Eng. 8 (2009) 635.

[3]. G. Madurambal, M. Mariyappan, S. Monojumdar, J. Therm. Anal. Calorim, 100 (2010)763.

[4]. S. B Monaco, L. E. Davis, S. P. Velsko, F. T. Wang, D. Eimerl, A Zalkin, J. Crystal Growth 85 (1987) 252.

[5]. $\quad$ L. N. Rashkovich, B.Y. Shekunov, J. Crystal Growth 112(1991) 183.

[6]. M.D. Aggarwal, J. Choi , W.S.Wang, K. Bhat, R.B. Lal, A.D.Shields, J Crystal Growth (204) 1999179.

[7]. D.Rajan Babu, D.Jayaraman, R.Mohankumar, R.Jayavel, J. Crystal Growth 245(2002)121.

[8]. $\quad$ R. Ittyachan, P. Sagayaraj, J. Crystal Growth 249 (2003) 557.

[9]. U. Chatterjee, A.M. Rudra, G.C. Bhar, Appl. Phys. B 61 (1995) 489.

[10]. F. Laeri, R. Jungen, G. Angelow, U. Vietze, T. Engel, M. Wuïrtz, D. Hilgenberg, Appl. Phys. B 61 (1995) 351.

[11]. V. Gopalan, M.C. Gupta, Appl. Phys. Lett. 68 (1996) 888.

[12]. M.R. Suresh Kumar, H.J. Ravindra, S.M. Dharmaprakash Journal of Crystal Growth 306 (2007) 361-365.

[13]. D. Joseph Daniel, P. Ramasamy, Optical Materials 36 (2014) 971.

[14]. A.S.J. Lucia Rose, P. Selvarajan, S. Perumal, Spectrochimica Acta Part A, 81 (2011) 270.

[15]. S.K. Kurtz, T.T. Perry, J. Appl. Phys. 39 (1968) 3798.

[16]. U. Von Hundelshausen, Phys. Lett. A 34 (1971) 405. 